\title{
Mission Overview and Initial Observation Results of the X-Ray Pulsar Navigation-I Satellite
}

\author{
Xinyuan Zhang, ${ }^{1}$ Ping Shuai, ${ }^{1}$ Liangwei Huang, ${ }^{1}$ Shaolong Chen, ${ }^{1}$ and Lihong $\mathrm{Xu}^{2}$ \\ ${ }^{1}$ Qian Xuesen Laboratory of Space Technology, Beijing 100094, China \\ ${ }^{2}$ China Academy of Space Technology, Beijing 100094, China \\ Correspondence should be addressed to Liangwei Huang; huangliangwei@qxslab.cn
}

Received 28 March 2017; Revised 21 May 2017; Accepted 7 June 2017; Published 6 July 2017

Academic Editor: Linda L. Vahala

Copyright (c) 2017 Xinyuan Zhang et al. This is an open access article distributed under the Creative Commons Attribution License, which permits unrestricted use, distribution, and reproduction in any medium, provided the original work is properly cited.

\begin{abstract}
The newly launched X-ray pulsar navigation-I (XPNAV-1) is an experimental satellite of China that is designed for X-ray pulsar observation. This paper presents the initial observation results and aims to recover the Crab pulsar's pulse profile to verify the Xray instrument's capability of observing pulsars in space. With the grazing-incidence focusing type instrument working at the soft $\mathrm{X}$-ray band ( $0.5-10 \mathrm{keV})$, up to 162 segments of observations of the Crab pulsar are fulfilled, and more than 5 million X-ray events are recorded. Arrival times of photons are corrected to the solar system barycentre, and the $33 \mathrm{~ms}$ pulse period is sought out for Crab. Epoch folding of all the corrected photon times generates the refined pulse profile of Crab. The characteristic two-peak profile proves that the Crab pulsar has been clearly seen, so that the conclusion is made that XPNAV-1's goal of being capable of observing pulsars is achieved.
\end{abstract}

\section{Introduction}

Pulsars [1], which are recognized as rotating neutron stars, can emit regular signals from the radio to the high energy band [2]. Navigation using X-ray pulsars [3-5] is regarded as a revolutionary technology providing autonomous spacecraft navigation capability in the whole solar system.

To demonstrate this technology, China Academy of Space Technology (CAST) has brought forward a three-step space demonstration scheme. The first step is the X-ray pulsar navigation-I (XPNAV-1) satellite, the core goal of which is to validate the capability of observing X-ray pulsars. The following step is to launch a medium-sized satellite in about $2 \sim 3$ years to accumulate more $\mathrm{X}$-ray data for pulsar parameter database construction and to test pulsar navigation algorithm onboard after 3 5 pulsars are timed enough accurately. The third step is to build a constellation system to demonstrate navigation application and time service using $\mathrm{X}$-ray pulsars.

XPNAV-1 was launched at Beijing time Nov. 10, 2016, by the Long March 11 rocket at the Jiuquan Satellite Launch Centre. It is a small satellite weighing about $270 \mathrm{~kg}$ and works on the $500 \mathrm{~km}$ LEO orbit. The purpose of the XPNAV-1 mission is to test the technology of pulsar observation in the soft X-ray band through the X-ray instruments developed by CAST. Three objectives are outlined: (1) test function and performance for the X-ray instruments in the outer space; (2) detect typical X-ray pulsars' radiation photons and acquire the pulse profiles to verify the ability of X-ray pulsar observation; (3) accumulate X-ray data for a long time to measure the pulsars' parameters via X-ray timing.

The XPNAV-1 satellite works at the three-axis stabilization attitude mode with the ability to quickly point to any inertial position according to demand as accurate as 2 arcminutes and to provide up to 90 minutes' sustained observation limited by the power supply. The satellite operates two X-ray devices. One is the Time-resolved soft Xray spectrometer (TSXS), and the other is the high timeresolution photon counter (HTPC). The TSXS uses a WolterI type lens of 4 nested mirror shells with the collecting area of $30 \mathrm{~cm}^{2}$ to focus X-ray photons within 15 arcminutes fieldof-view onto a silicon drift detector (SDD). A GPS calibrated $\mathrm{Rb}$ clock is included to provide accurate time and a quasiparallelly optical star is used to assist the inertial pointing. The TSXS device provides the $1.5 \mu$ s time resolution and the 
TABLE 1: Default targets' angular positions and pulse periods.

\begin{tabular}{lcccc}
\hline Number & Pulsar name & J2000 right ascension $(\mathrm{deg})$ & J2000 declination (deg) & Pulse period (ms) \\
\hline 1 & B0531+21 (Crab) & 83.63303 & 22.01449 & 33.085 \\
2 & B1617-155 (Sco X-1) & 244.979 & -15.640 & 3.200 \\
3 & B1758-250 & 270.284 & -25.079 & 3.000 \\
4 & B1813-140 & 274.006 & -14.036 & 3.300 \\
5 & GROJ1744-28 & 266.138 & -28.741 & 467.000 \\
6 & B0540-69 & 85.04668 & -69.33171 & 50.499 \\
7 & B1509-58 & 228.48175 & -59.13583 & 150.658 \\
8 & J1846-0258 & 281.60392 & -2.97503 & 325.684 \\
\hline
\end{tabular}

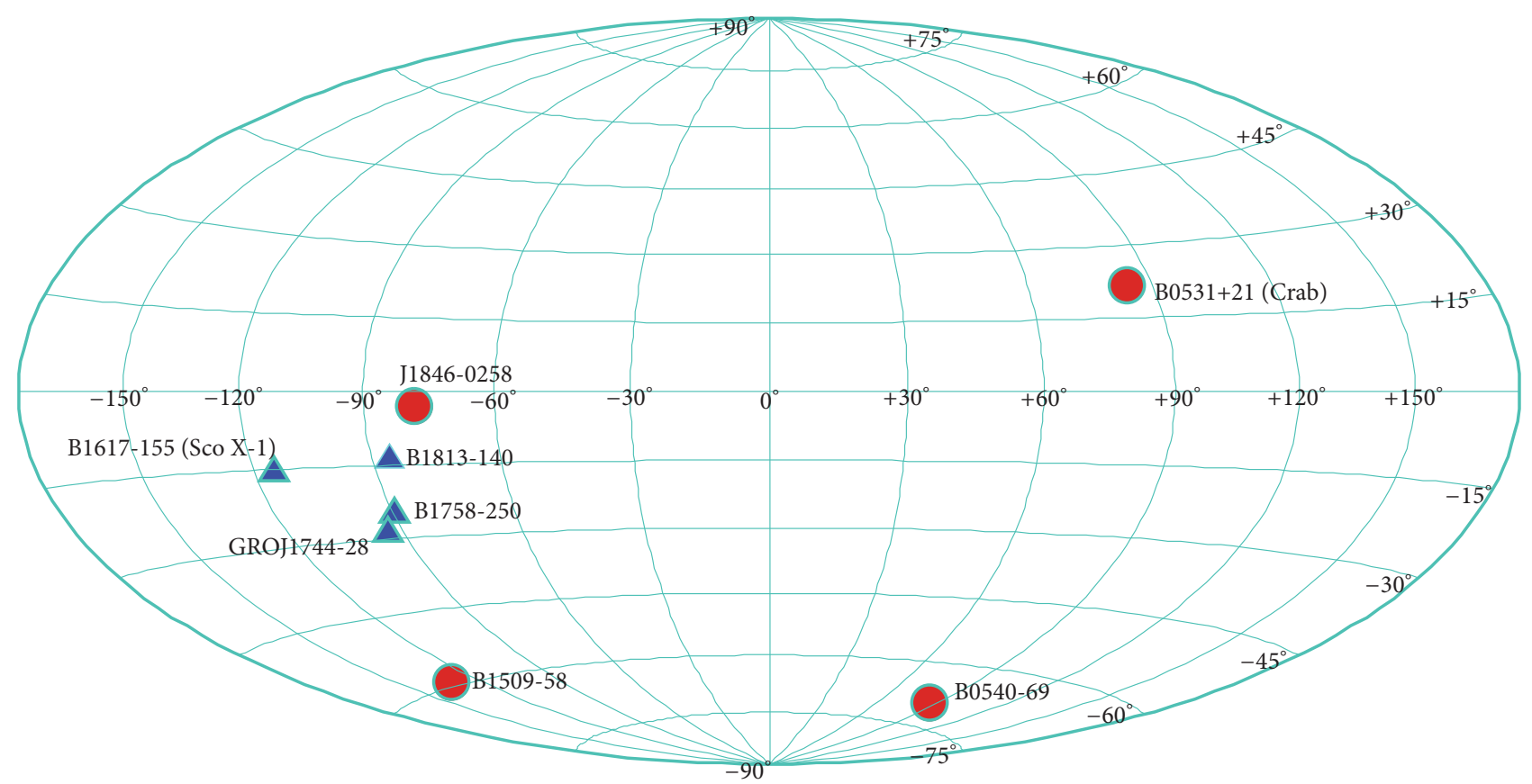

IRP
XB

FIGURE 1: Default targets' angular positions plotted in the J2000 frame.

$180 \mathrm{eV} @ 5.9 \mathrm{keV}$ energy resolution in the $0.5 \sim 10 \mathrm{keV}$ energy band. The HTPC device uses the collimator to confine the field-of-view to 2 degrees and the microchannel plate (MCP) $\mathrm{X}$-ray detector to count the X-ray photons in the $1 \sim 10 \mathrm{keV}$ energy band from the pulsar. Compared to the TSXS, the MCP of the HTPC has a higher time resolution of $100 \mathrm{~ns}$ and a bigger collecting area of $1200 \mathrm{~cm}^{2}$.

In order to examine the satellite-borne X-ray instruments, we choose the Crab pulsar [6] as the calibration target because this pulsar is believed to be one of the best studied objects in the sky and one of the brightest X-ray sources regularly studied. Current data show that TSXS exhibits a good and steady performance in X-ray photon collection. In this paper, we will present the data analysis results of the TSXS observation on Crab as well as the mission overview. We organize this paper as follows. The mission flight system is described in Section 2. The XPNAV-1 Xray data analysis software system is introduced in Section 3. The observations and the data analysis results are provided in Section 4. The conclusions and discussions are made in Section 5.

\section{XPNAV-1 Mission Overview}

The core objective of the XPNAV-1 mission is to validate the ability of observing X-ray pulsars. To achieve this, eight Xray sources are chosen as default targets. Not only 4 isolated rotation-powered (IRP) pulsars but also $4 \mathrm{X}$-ray binaries (XB) are included because of their brightness. The eight sources' angular positions and pulse periods [7-9] are shown in Table 1 and Figure 1. XPNAV-1 provides four observation modes: the self-test mode, the scanning mode, the default target 


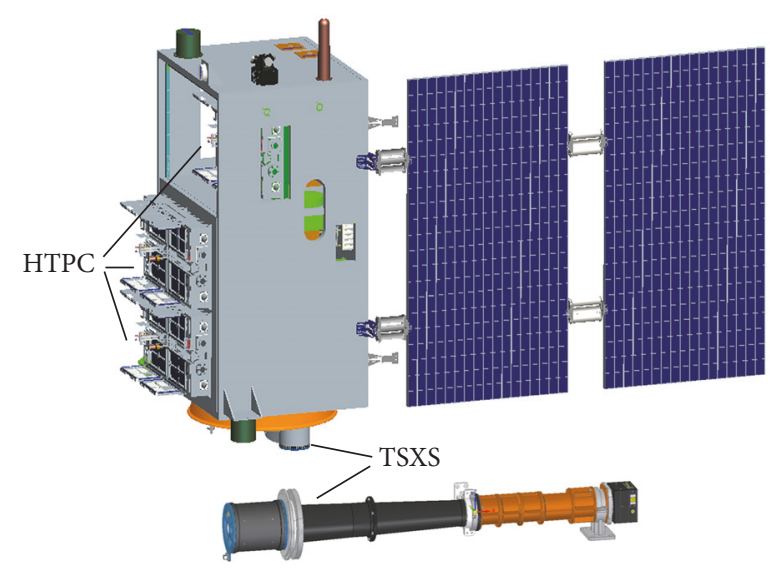

FIGURE 2: XPNAV-1 satellite structure overview.

observation mode, and the arbitrary target pointing mode. The self-test mode works when the lens is covered with the hood in order that the instrument noise can be measured. The scanning mode is to scan a belt of the space via rotating the satellite in order to evaluate the background noise. The default target observation mode makes the X-ray instrument point at one of the 8 default targets. The arbitrary target pointing mode is similar to the former mode except that the angular position parameters should be uploaded from ground.

Figure 2 provides an overview of the XPNAV-1 satellite structure. The satellite applies integrated electronics design, with which the functions of onboard data handling $(\mathrm{OBDH})$, tracking telemetry \& command (TTC), attitude determination and control, GNSS navigation, and power control are integrated in one electronic component. A zero momentum three-axis attitude control system is exploited with highprecision inertial pointing capability.

The XPNAV-1 satellite operates on a sun-synchronous orbit, of which the orbit semimajor axis is $6878.137 \mathrm{~km}$, the inclination is 97.4 degrees, and the local time of descending node (LTDN) is 6:00 AM. Two instruments, TSXS and HTPC, are mounted along different directions. When the observation carries on, the satellite spins to make the X-ray instrument inertially pointing at the target, and when the observation is finished, the satellite spins to make the solar array point to the sun. The two instruments cannot work simultaneously because of the insufficient capability of the power supply.

In Beijing time Nov. 17, 2016, XPNAV-1 fulfilled the satellite test and entered the observation phase. Until Feb., 2017, XPNAV-1 has observed three sources, that is, PSR B0531+21 (Crab), PSR B0540-69, and PSR B1509-58. During each observation, $\mathrm{X}$-ray devices are shut down in the region of south Atlantic anomaly (SAA) to avoid the potentially harmful impact on the working detector. During the Earth occultation, no observation is planned. Besides, when the angle between the target and the sun or the moon is less than
45 degrees, observation is ceased to avoid the interference signal.

The preliminary data show that TSXS has steady performance in the signal-to-noise ratio. Thus, we implement most observations on the Crab pulsar using TSXS. Totally, 162 TSXS observations of Crab are performed, each of which lasts 10 to 90 minutes. In the following sections, we will introduce the data analysis software system and present the analysis results.

\section{Data Analysis Software System}

The data analysis software system (DASS) is constructed to process XPNAV-1 data. The main purpose of DASS is to acquire the pulsar's pulse profile from the observed X-ray data in order to testify to the X-ray instrument's capability of observing pulsars. The system consists of nine parts, each of which is designed as a functional software. The nine pieces of software are separately named as ORIDATA, XSELECT, XFLUX, XENERGY, ORBITPROP, XCORR, NOMDB, $X F S E A R C H$, and $X F O L D$, which are organized as Figure 3 shows.

ORIDATA deals with the original data unpacking. The telemetry, platform, and payload data transmitted from the satellite are unpacked and regrouped into the GPS data, the onboard orbit data, the satellite status flag data, the satellite attitude data, and the X-ray photon data. XSELECT performs X-ray photon selection according to the satellite status flag and the satellite attitude. The photons at the time when the satellite works well and the X-ray instrument points accurately to the source remained. XFLUX counts the photons to calculate the source or the background flux. $X E N E R G Y$ analyzes the photon energy data for the spectrum and radiation model researches. ORBITPROP propagates the orbit to determine the satellite's inertial position at every single photon's arrival time from the GPS data or the onboard orbit data. XCORR corrects the photon arrival time at the satellite to the arrival time at the solar system barycentre (SSB) or the emission time at the pulsar [10-14]. NOMDB [15] is the nominal pulsar parameter database, which provides the current known pulsar parameters for the data handling. $X F S E A R C H$ searches for the pulse period and its derivatives from the corrected photon arrival times in the time domain as well as the frequency domain $[16,17]$, and it will update the nominal database's pulsar parameters if necessary. XFOLD generates the pulsar's profile through epoch folding [18] and analyze its timing property.

\section{Observations and Results}

From coordinate universal time (UTC) 57709.0 in modified Julian date (MJD) to 57872.0 MJD, TSXS carried out 162 segments of observations upon PSR B0531+21. These TSXS observation data are made public and can be downloaded at the Beidou official website [19]. The data analysis work in this section is performed using this set of public data. The mean duration of the observations is about 39 minutes. From these observations, 5824511 photons in the $0.5 \sim 10 \mathrm{keV}$ band 


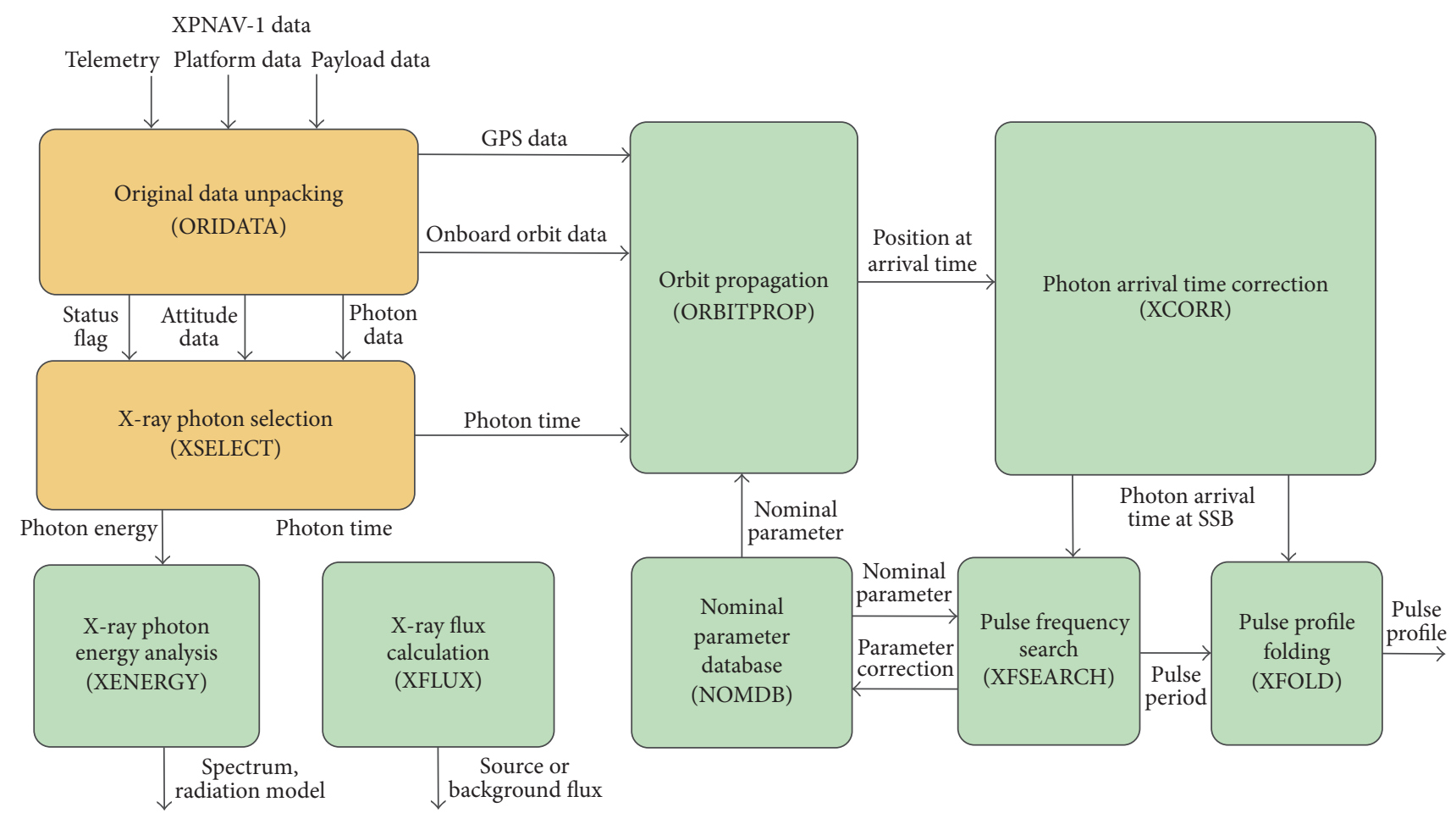

Figure 3: Structure and data flow of the data analysis software system.
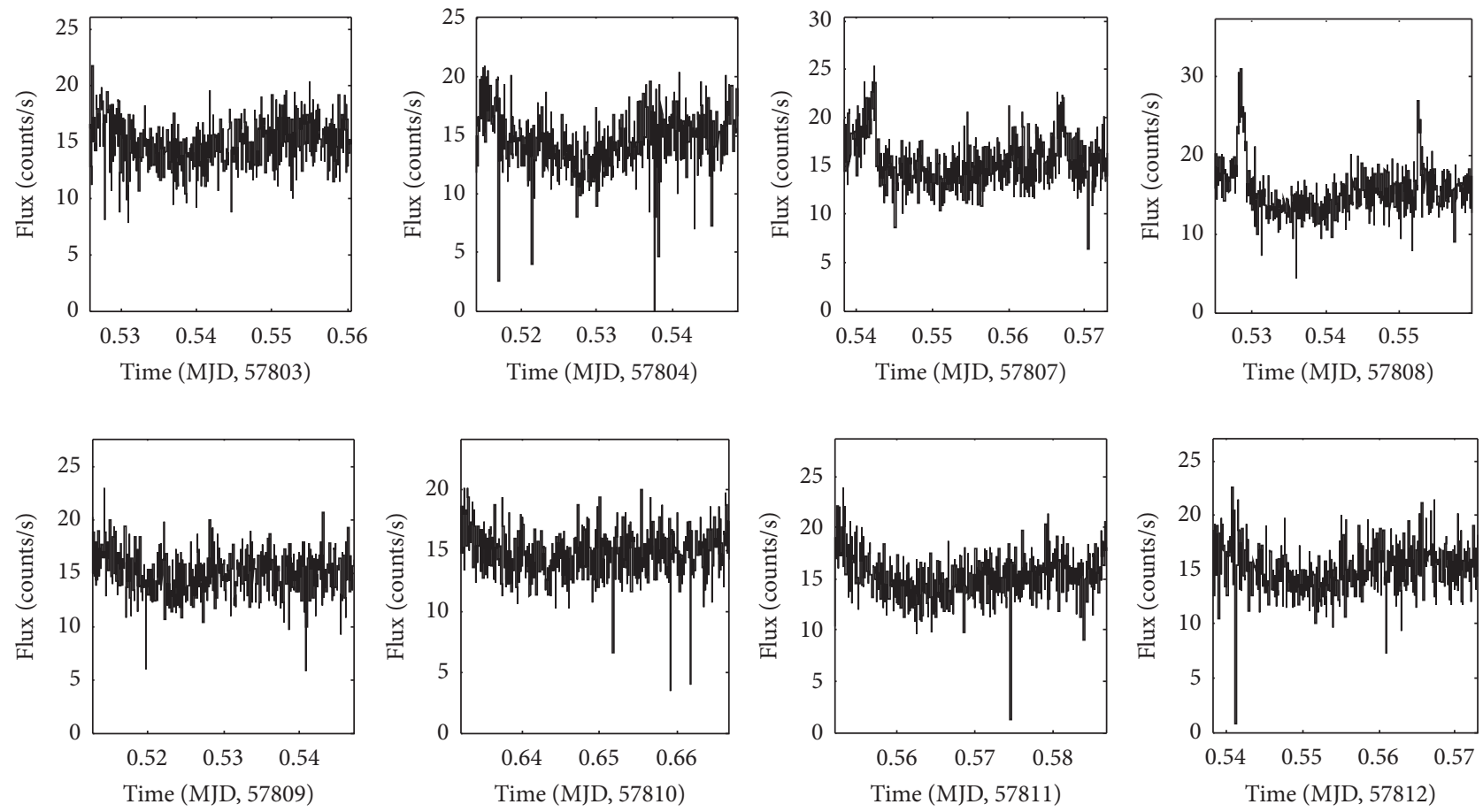

FIGURE 4: TSXS flux data of eight observations on Crab.

are collected. The mean flux in this band is 15.4 counts/s. The flux data of eight observations from UTC 57803.5 MJD to UTC 57812.6 MJD are illustrated in Figure 4.

The derived spectrum of $\mathrm{Crab}$ in the $0.5 \sim 7 \mathrm{keV}$ band is shown in Figure 5. The vertical line in the figure represents the 3-sigma error. The spectrum analysis and the calibration of TSXS's energy property with respect to the radiation model will be our future work.

The photon arrival times at the X-ray device are corrected to the solar system barycentre (SSB) arrival times via the 


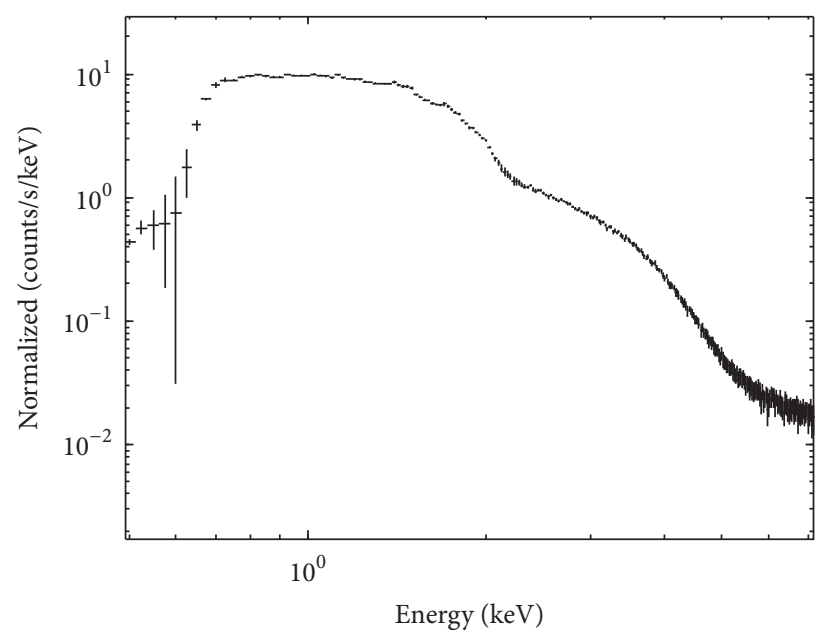

FIGURE 5: Spectrum of Crab in the $0.5 \sim 7 \mathrm{keV}$ band from TSXS.
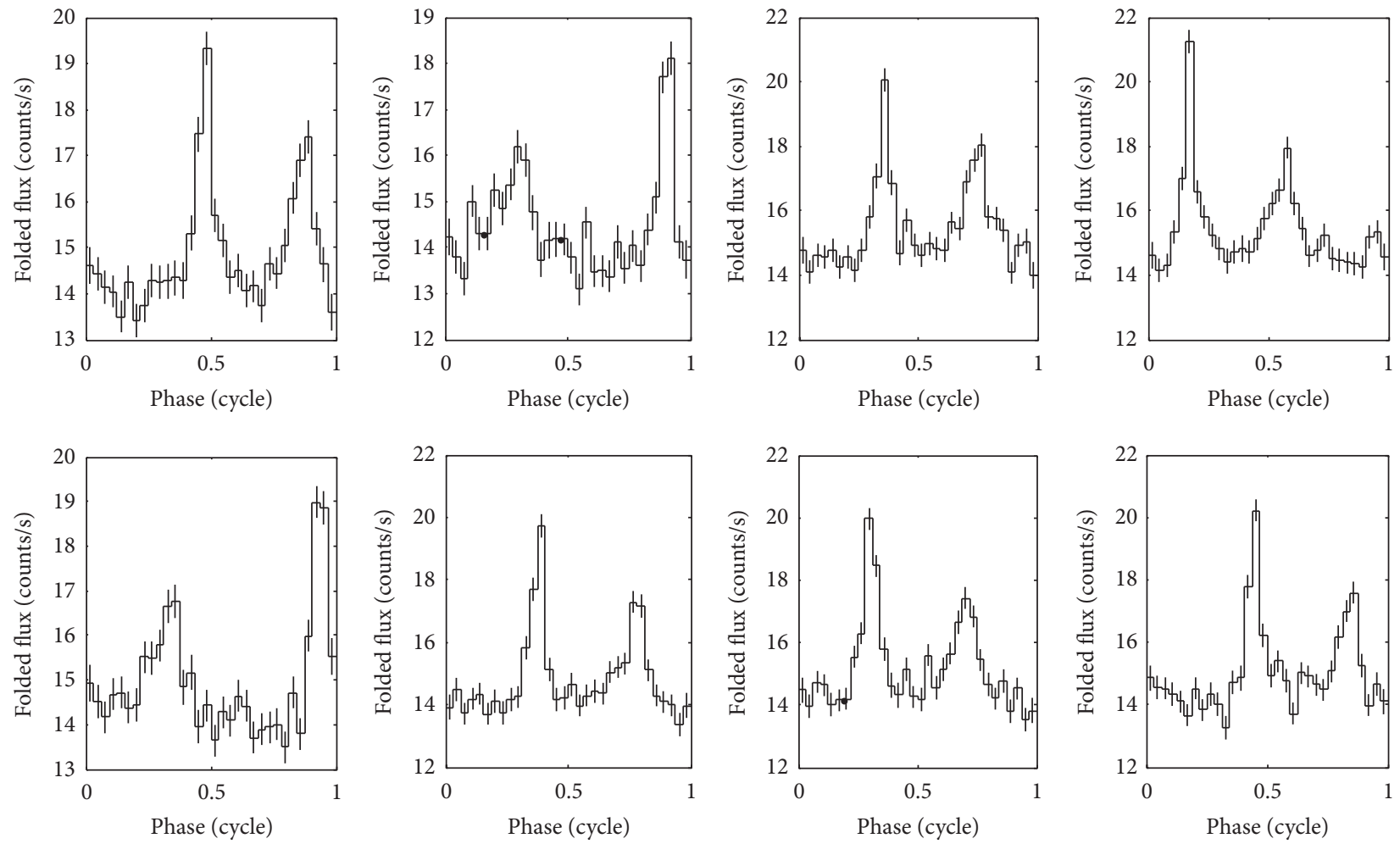

FIGURE 6: Crab profiles in the 0.5 9 keV band from eight observations from UTC 57803.5 MJD to UTC 57812.6 MJD.

orbit data. The periodicity is found from the corrected times in the frequency domain, and then the accurate pulse period and its derivatives are searched for in the time domain. With the period parameters, the pulse profile can be acquired by epoch folding from each observation. Furthermore, all observations can be combined to form a much finer profile.

In Figure 6, we provide eight folded Crab profiles in the $0.5 \sim 9 \mathrm{keV}$ band derived from the corresponding observations in Figure 4. The profiles use 32 bins, the vertical lines of which indicate the folded 1 sigma folding flux error. The zero phase time in Figure 6 is taken as the observation beginning time. Therefore, by shifting the profile [20], the pulse time of arrival (TOA) corresponding to the peak time can be accurately figured out so that 162 pulse TOAs are acquired.

The combined Crab profile in the $0.5 \sim 9 \mathrm{keV}$ band is plotted in Figure 7. This profile uses 512 phase bins, the characteristic two peaks [21] of which kept apart at about 0.4 cycles are clearly identified. The zero phase of the plot is aligned to the main peak. From this profile, the pulsed fraction of the Crab radiation in the $0.5 \sim 9 \mathrm{keV}$ band can be estimated to be $5.3 \%$. 


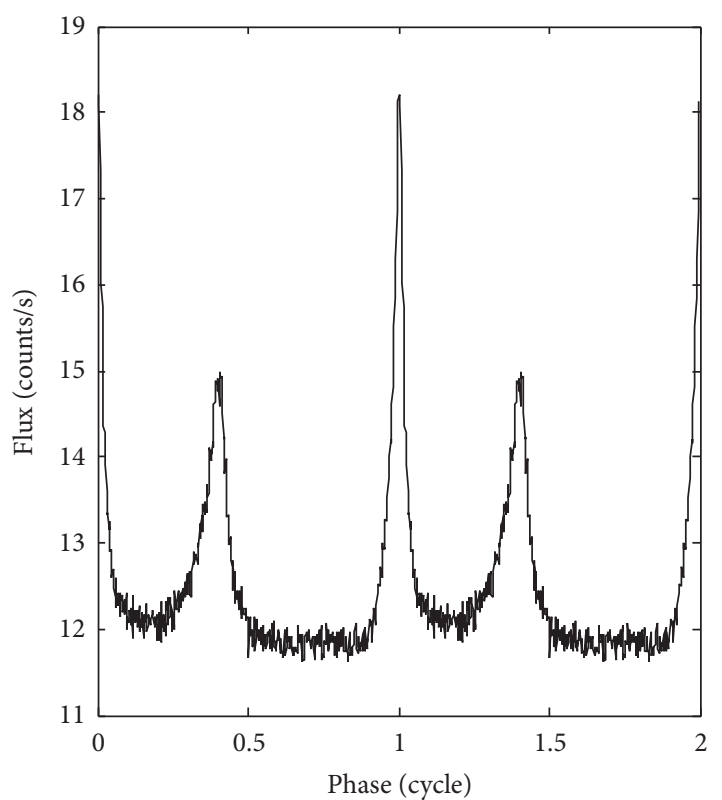

Figure 7: Crab profiles in the $0.5 \sim 9 \mathrm{keV}$ band from all 162 observations.

\section{Conclusions and Discussion}

In this paper, we give an overview of the XPNAV-1 mission, the properties of the X-ray instruments, and the data analysis software system. The TSXS observations on Crab are described in detail. Up to 162 segments of observations are collected containing over 5 million X-ray photons with a coming rate at 15.4 counts/s on average. Arrival times of photons within $0.5 \sim 9 \mathrm{keV}$ are corrected to SSB and epochfolded, which produces folded flux curves corresponding to 162 pulse TOAs. Combination of the observations generates the refined profile for Crab, which shows a $5.3 \%$ pulsed fraction in the $0.5 \sim 9 \mathrm{keV}$ band. The characteristic profile of Crab has validated XPNAV-l's capability of observing pulsars in the soft X-ray band, which implies that the aim of the XPNAV-1 mission has been achieved. Next, we will research on how to use the Crab observation data to assist the orbit determination. Although three-dimensional positioning cannot be realized by observing only one pulsar, the divergence of the orbit propagation could be slowed down by incorporating the radial range measurement along Crab's line-of-sight. We will also spend more time observing the weaker sources and explore the possibility of navigation if more pulsars are observed. XPNAV-1 satellite is the first step of the consecutive missions designed by CAST for the demonstration of X-ray pulsar navigation. Next mission will bring to space an X-ray detector with a much larger collection area with the hope to accurately time $3 \sim 5$ pulsars and fulfill the onboard navigation.

\section{Conflicts of Interest}

The authors declare that there are no conflicts of interest regarding the publication of this paper.

\section{Acknowledgments}

This work was supported by the National Natural Science Foundation of China (Grants nos. 61403391, 61601463, and 11405265).

\section{References}

[1] A. Hewish, S. J. Bell, J. D. H. Pilkington, P. F. Scott, and R. A. Collins, "Observation of a rapidly pulsating radio source," Nature, vol. 217, no. 5130, pp. 709-713, 1968.

[2] R. D. Lorimer and M. Kramer, Handbook of Pulsar Astronomy, Cambridge Univ Press, Cambridge, UK, 2005.

[3] E. Hanson J, Principles of X-ray navigation. Ph.D Dissertation, [Ph.D. thesis], Dept of Aeronautics and Astronautics, Stanford Univ, Stanford, Calif, USA, 1996.

[4] S. I. Sheikh, D. J. Pines, P. S. Ray, K. S. Wood, M. N. Lovellette, and M. T. Wolff, "Spacecraft navigation using X-ray pulsars," Journal of Guidance, Control, and Dynamics, vol. 29, no. 1, pp. 49-63, 2006.

[5] P. Shuai, M. Li, and L. S. Chen, Principles and Methods for X-ray Pulsar-based navigation System, China Astronautic Publishing House, Beijing, China, 2009.

[6] M. G. F. Kirsch, U. G. Briel, D. Burrows et al., "Crab: The standard X-ray candle with all (modern) X-ray satellites," in Proceedings of the UV, X-Ray, and Gamma-Ray Space Instrumentation for Astronomy XIV, SPIE, pp. 1-12, August 2005.

[7] R. N. Manchester, G. B. Hobbs, A. Teoh, and M. Hobbs, "The Australia telescope national facility pulsar catalogue," Astronomical Journal, vol. 129, no. 4, pp. 1993-2006, 2005.

[8] The Australia Telescope National Facility (ANTF) Pulsar Catalogue. 2011, available: http://www.atnf.csiro.au/research/pulsar/ psrcat.

[9] S. I. Sheikh, The use of variable celestial X-ray sources for spacecraft navigation [Ph.D. thesis], Dept Aero Eng, Maryland Univ, College Park, MD, USA, 2005.

[10] T. Damour and N. Deruelle, "General relativistic celestial mechanics of binary systems. I. The post-Newtonian motion," Annales de l'Institut Henri Poincaré (Physicque théorique), vol. 43, no. 1, pp. 107-132, 1985.

[11] T. Damour and N. Deruelle, "General relativistic celestial mechanics of binary systems II. The post-Newtonian timing formula," Annales de l'Institut Henri Poincaré (Physicque théorique), vol. 44, no. 3, pp. 263-292, 1986.

[12] G. B. Hobbs, R. T. Edwards, and R. N. Manchester, "TEMPO2, a new pulsar-timing package - I. An overview," Monthly Notices of the Royal Astronomical Society, vol. 369, no. 2, pp. 655-672, 2006.

[13] R. T. Edwards, G. B. Hobbs, and R. N. Manchester, "TEMPO2, a new pulsar timing package - II. The timing model and precision estimates," Monthly Notices of the Royal Astronomical Society, vol. 372, no. 4, pp. 1549-1574, 2006.

[14] L. Huang, B. Liang, T. Zhang, and C. Zhang, "Navigation using binary pulsars," Science China: Physics, Mechanics and Astronomy, vol. 55, no. 3, pp. 527-539, 2012.

[15] L. Huang, P. Shuai, Q. Lin, and X. Zhang, "Research on nominal database for X-ray pulsar navigation," Chinese Space Science and Technology, vol. 35, no. 3, pp. 66-74, 2015.

[16] X.-Y. Zhang, P. Shuai, and L.-W. Huang, "Profile folding distortion and period estimation for pulsar navigation," Journal of Astronautics, vol. 36, no. 9, pp. 1056-1060, 2015. 
[17] Y. Q. Zhou, F. J. Ji, and H. F. Ren, "Quick search algorithm of Xray pulsar period based on unevenly spaced timing data," Acta Physica Sinica, vol. 62, no. 1, pp. 1-8, 2013.

[18] A. A. Emadzadeh and J. L. Speyer, "X-ray pulsar-based relative navigation using epoch folding," IEEE Transactions on Aerospace and Electronic Systems, vol. 47, no. 4, pp. 2317-2328, 2011.

[19] XPNAV-1 Observation Data, 2017, http://www.beidou.gov.cn/ xpnavdata.rar.

[20] A. A. Emadzadeh and J. L. Speyer, "On modeling and pulse phase estimation of X-ray pulsars," IEEE Transactions on Signal Processing, vol. 58, no. 9, pp. 4484-4495, 2010.

[21] MPIfR EPN Pulsar Profiles Database, 2017, http://rian.kharkov .ua/decameter/EPN/browser.html. 


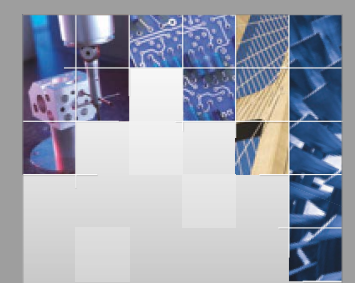

\section{Enfincering}
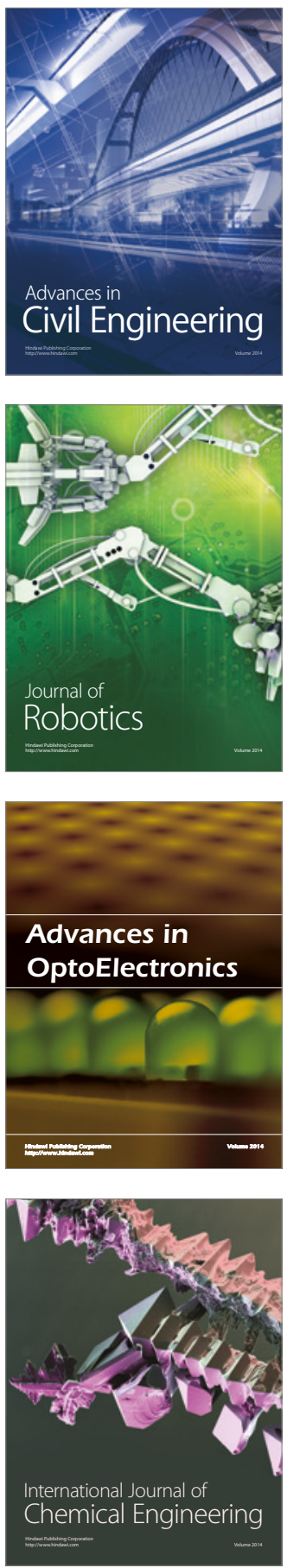

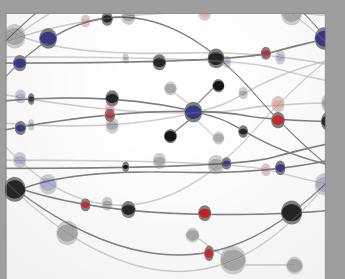

The Scientific World Journal

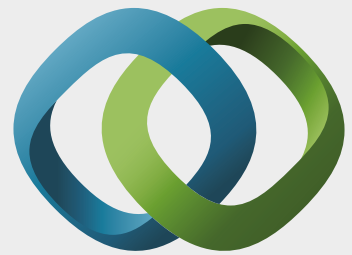

\section{Hindawi}

Submit your manuscripts at

https://www.hindawi.com
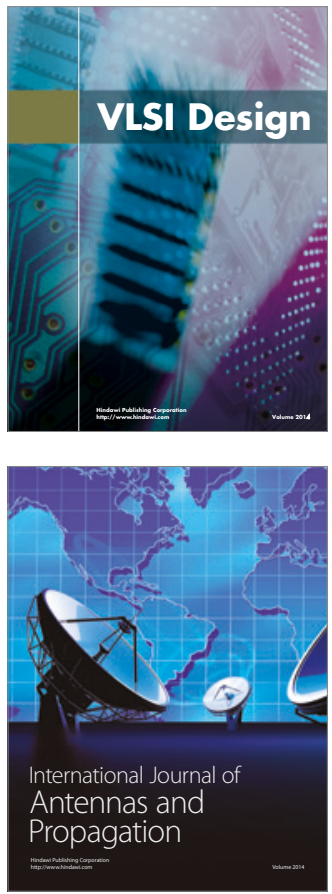

\section{Rotating}

Machinery
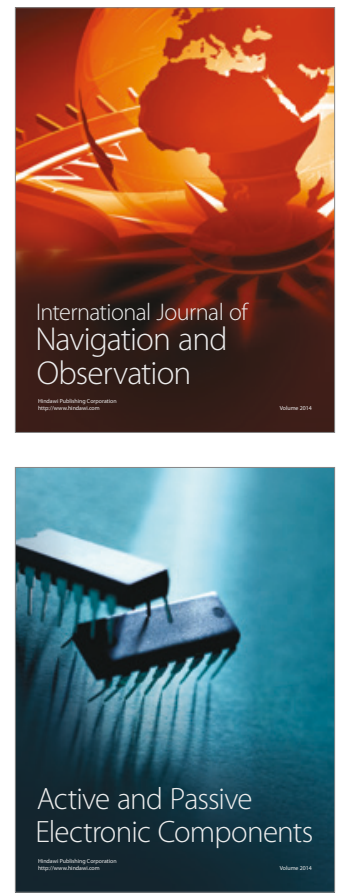
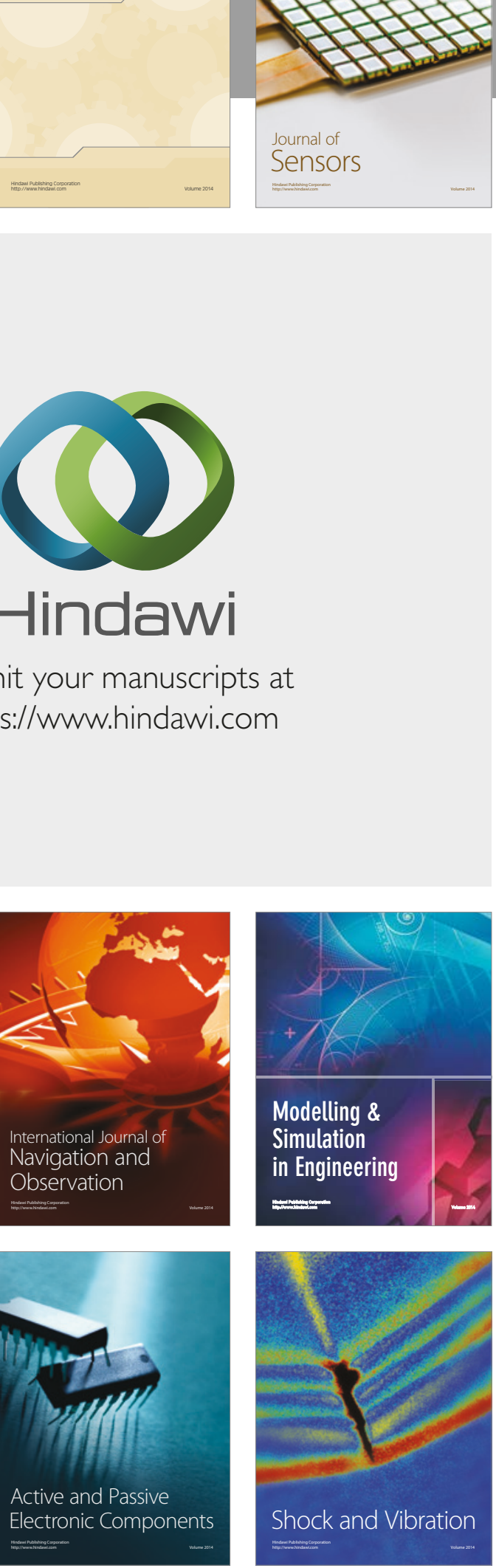
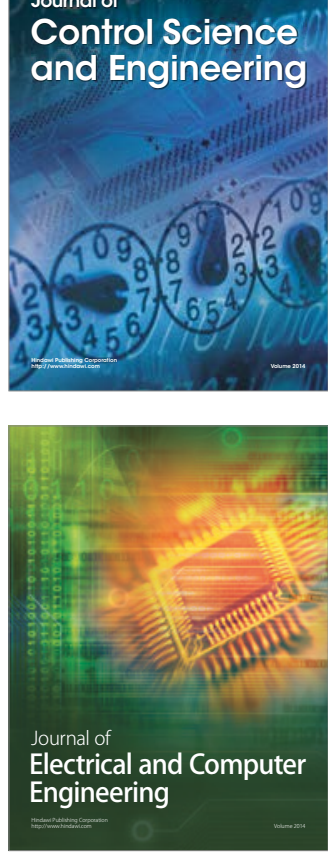

Distributed

Journal of

Control Science

and Engineering
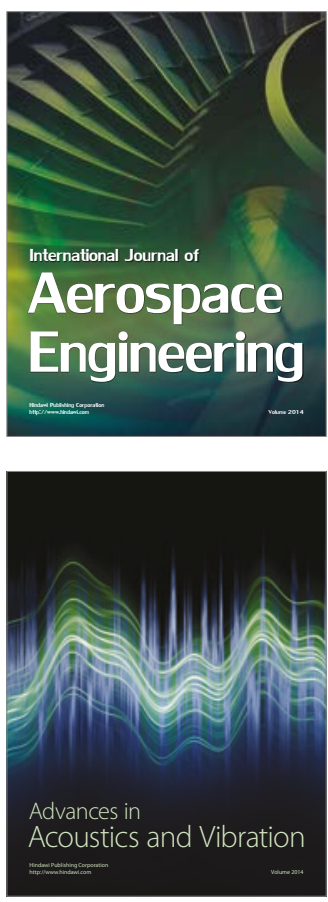

Sensor Networks 